\title{
A Histologic Scoring System for Prognosis of Patients With Alcoholic Hepatitis
}

\author{
José Altamirano, $\mathbf{M D}^{1}$, Rosa Miquel, $\mathbf{M D}^{2}$, Aezam Katoonizadeh, $\mathbf{M D}^{3}$, Juan G Abraldes, \\ MD $^{4,12}$, Andrés Duarte-Rojo, MD ${ }^{5,15}$, Alexandre Louvet, MD $^{6}$, Salvador Augustin, MD ${ }^{7}$, \\ Rajeshwar P. Mookerjee, MD ${ }^{8}$, Javier Michelena, MD ${ }^{1}$, Thomas C. Smyrk, MD ${ }^{9}$, David Buob, \\ MD ${ }^{10}$, Emmanuelle Leteurtre, MD ${ }^{10}$, Diego Rincón, MD ${ }^{11}$, Pablo Ruiz, MD ${ }^{1}$, Juan Carlos \\ García-Pagán, MD ${ }^{1,12}$, Carmen Guerrero-Marquez, MD ${ }^{13}$, Patricia D. Jones ${ }^{14}$, A. Sidney \\ Barritt IV, MD ${ }^{14}$, Vicente Arroyo, MD ${ }^{1}$, Miquel Bruguera, MD1 , Rafael Bañares, MD ${ }^{11}$, Pere \\ Ginès, MD ${ }^{1}$, Juan Caballería, MD ${ }^{1}$, Tania Roskams, MD³ , Frederik Nevens, MD, Rajiv Jalan, \\ MD ${ }^{8}$, Philippe Mathurin, MD ${ }^{6}$, Vijay H. Shah, MD ${ }^{5}$, and Ramón Bataller, MD ${ }^{1,14}$ \\ ${ }^{1}$ Liver Unit, Hospital Clinic, Institut d'Investigacions Biomèdiques August Pi i Sunyer (IDIBAPS), \\ Barcelona, Spain. CIBER de Enfermedades Hepáticas y Digestivas (CIBERehd) \\ ${ }^{2}$ Pathology Unit, Hospital Clinic, Institut d'Investigacions Biomèdiques August Pi i Sunyer \\ (IDIBAPS), Barcelona, Spain. CIBER de Enfermedades Hepáticas y Digestivas (CIBERehd) \\ ${ }^{3}$ Department of Morphology and Molecular Pathology, University Hospital Gasthuisberg, KU \\ Leuven, Leuven, Belgium \\ ${ }^{4}$ Division of Gastroenterology, Department of Medicine. University of Alberta, Edmonton, Canada \\ ${ }^{5}$ Division of Gastroenterology and Hepatology, Mayo Clinic, Rochester, MN \\ ${ }^{6}$ Services d'Hepáto-Gastroentérologie, CHRU, Université de Lille. EA2686, Faculté de Médecine \\ Pôle Recherche and Inserm U837 Lille, France. Hôpital Huriez Lille, France \\ ${ }^{7}$ Liver Unit, Department of Internal Medicine, Hospital Universitari Vall d'Hebron, Institut de \\ Recerca (VHIR), Universitat Autònoma de Barcelona, Barcelona, Spain \\ ${ }^{8}$ UCL Institute of Hepatology, Royal Free Hospital, London, UK
}

(C) 2014 The American Gastroenterological Association. Published by Elsevier Inc. All rights reserved.

Address for correspondence: Ramón Bataller, M.D. PhD., Associate Professor, Departments of Medicine and Nutrition, 7340 Medical Biomolecular Research Building, University of North Carolina at Chapel Hill, Chapel Hill, NC 27599, Tel 919-966-4812, bataller@med.unc.ed.

CONFLICTS OF INTEREST: The authors disclose no conflicts.

AUTHOR'S CONTRIBUTION: Study concept, design, collection and interpretation of data and histological analysis: José Altamirano, Rosa Miquel and Ramón Bataller. Acquisition of data and histological analysis: Aezam Katoonizadeh, David Buob, Emmanuelle Leteurtre, Carmen Guerrero-Marquez and Thomas C. Smyrk. Generation and collection of data: Andrés Duarte-Rojo, Alexandre Louvet, Javier Michelena, Rajeshwar P. Mookerjee, Pablo Ruiz and Diego Rincón. Drafting of the manuscript: Jose Altamirano and Ramón Bataller. Critical revision and statistical analysis: Juan G Abraldes, Salvador Augustin, Patricia D. Jones and Jose Altamirano. Critical revision of the original draft: Miquel Bruguera, Rafael Bañares, Pere Ginès, Juan Caballería, Tania Roskams, Frederik Nevens, Rajiv Jalan, Philippe Mathurin, Vijay H. Shah, A. Sidney Barritt IV, Vicente Arroyo and Juan Carlos García-Pagán. All authors review and approved the final version of the manuscript.

Publisher's Disclaimer: This is a PDF file of an unedited manuscript that has been accepted for publication. As a service to our customers we are providing this early version of the manuscript. The manuscript will undergo copyediting, typesetting, and review of the resulting proof before it is published in its final citable form. Please note that during the production process errors may be discovered which could affect the content, and all legal disclaimers that apply to the journal pertain. 
${ }^{9}$ Department of Pathology. Mayo Clinic, Rochester, MN

${ }^{10}$ Institut de Pathologie, CHRU, Université de Lille. EA2686, Faculté de Médecine Pôle Recherche and Inserm U837 Lille, France. Hôpital Huriez Lille, France

${ }^{11}$ Liver Unit, Gastroenterology and Hepatology Division, Hospital General Universitario Gregorio Marañon, IISGM, School of Medicine, Universidad Complutense, Madrid, Spain. CIBER de Enfermedades Hepáticas y Digestivas (CIBERehd)

${ }^{12}$ Hepatic Hemodynamic Laboratory, Hospital Clinic, Institut d'Investigacions Biomèdiques August Pi i Sunyer (IDIBAPS), Barcelona, Spain. CIBER de Enfermedades Hepáticas y Digestivas (CIBERehd)

${ }^{13}$ Pathology Department, Hospital General Universitario Gregorio Marañon, IISGM, School of Medicine, Universidad Complutense, Madrid, Spain. CIBER de Enfermedades Hepáticas y Digestivas (CIBERehd)

${ }^{14}$ Division of Gastroenterology and Hepatology. Departments of Medicine and Nutrition. University of North Carolina at Chapel Hill, Chapel Hill, NC

${ }^{15}$ Division of Gastroenterology and Hepatology, University of Arkansas for Medical Sciences, Little Rock, AK

\section{Abstract}

Background \& Aims-There is no histologic classification system to determine prognoses of patients with alcoholic hepatitis (AH). We identified histologic features associated with disease severity and created a histologic scoring system to predict short-term (90 day) mortality.

Methods-We analyzed data from 121 patients admitted to the Liver Unit (Hospital Clinic, Barcelona, Spain) from January 2000 through January 2008 with features of AH, and developed a histologic scoring system to determine risk of death using logistic regression. The system was tested and updated in a test set of 96 patients from 5 academic centers in the US and Europe, and a semi-quantitative scoring system was developed, called the alcoholic hepatitis histologic score (AHHS). The system was validated in an independent set of 109 patients. Inter-observer agreement was evaluated by weighted statistic analysis.

Results-Degree of fibrosis, neutrophil infiltration, type of bilirubinostasis, and presence megamitochondria were independently associated with 90 day mortality. We used these 4 parameters to develop the AHHS to identify patients with low ( $0-3$ points), moderate (4-5 points), and high (69 points) risks of death within 90 days (3\%, 19\%, and 51\%, respectively; $P<.0001)$. The AHHS estimated 90 day mortality in the training and test sets with an area under the receiver operating characteristic value of 0.77 (95\% confidence interval, 0.71-0.83). Inter-rate agreement values were 0.65 for fibrosis, 0.86 for bilirubinostasis, 0.60 for neutrophil infiltration, and 0.46 for megamitochondria. Interestingly, the type of bilirubinostasis predicted the development of bacterial infections.

Conclusions-We identified histologic features associated with severity of AH and developed a patient classification system that might be used in clinical decision making. 


\section{Keywords}

alcoholic hepatitis; alcoholic liver disease; histological classification; liver biopsy

Alcoholic hepatitis (AH) is the most severe form of alcoholic liver disease (1). In its most severe form, short-term mortality of AH remains high (20-30\%) probably due to poor patient characterization and the need for modern targeted therapies (2). Current therapies (i.e. corticosteroids and pentoxifylline) fail in many patients and there is a clear need to develop new pathophysiologically-oriented approaches. Although the presence of AH can be suspected based on clinical and biochemical data, a definitive diagnosis requires histological confirmation (3). Regretfully, there are no well-validated non-invasive methods to establish the diagnosis of $\mathrm{AH}$.

In contrast to the huge burden of alcoholic liver disease and the prevalence of $\mathrm{AH}$, the research investment into this disease has been limited (4). Although prognosis can be estimated based on biochemical parameters $(2,5-7), \mathrm{AH}$ is probably the only prevalent liver disease that lacks a well-validated histological classification that can provide both prognostic and clinically meaningful data. Identifying key histological parameters associated with patient outcome can inform translational studies and also reveal key disease drivers. Histological classification schemes exist for other prevalent liver diseases such as chronic viral hepatitis, non-alcoholic steatohepatitis, autoimmune hepatitis and primary biliary cirrhosis (8-12). The present study attempts to fill an important gap in the field of AH. We performed a large multicentric study, including some of the main experts in this field, to develop a novel histological classification. Study, testing, and validation cohorts of patients with biopsy proven $\mathrm{AH}$ were included.

There are some reports showing that isolated histological findings can reflect disease severity in patients with AH (13-17). However, no studies have integrated these parameters to develop a meaningful histological classification. Thus, the aims of this study were to investigate how the interaction of individual histological features of AH predicts survival, and if it can be used to generate and validate a novel histological scoring system.

\section{METHODS}

\section{Study Cohort}

One hundred and eighty one consecutive patients admitted to the Liver Unit (Hospital Clinic, Barcelona, Spain) from January 2000 to January 2008 with clinical and analytical parameters suggesting an episode of $\mathrm{AH}$ were studied. The inclusion criteria were: excessive alcohol consumption (>60 g/day) prior to admission, moderately elevated aminotransferases with aspartate aminotransferase [AST] > alanine aminotransferase [ALT], high -glutamyl transpeptidase (GGT) and serum bilirubin levels, and a histological diagnosis of AH characterized by the presence of hepatocellular damage (hepatocellular ballooning and presence of Mallory bodies), inflammatory infiltrate (predominantly polymorphonuclear cells), and pericellular fibrosis $(2,18)$. All patient that gave an informed consent underwent a liver biopsy within 48 hours of admission as part of our clinical protocol. Sixty patients were 
not included due to other causes of liver disease (concomitant hepatitis $C$ virus $n=16$, concomitant hepatitis $C$ virus/HIV co-infection $n=3$, drug-induced hepatotoxicity $n=4$, hemochromatosis $n=1$, military tuberculosis $n=1$ and syphilitic hepatitis $n=1$ ) or incomplete histological criteria of $\mathrm{AH}(\mathrm{n}=34)$. After histological confirmation and a strict evaluation of inclusion and exclusion criteria, 121 patients with biopsy-proven AH were included and conformed the training set.

Severe AH was defined as a Maddrey's discriminant function $>32$ and/or an ABIC score $\checkmark 6.71$ at admission (2). Patients with Maddrey's discriminant function $>32$ were treated with prednisone orally for 4 weeks followed by a 2 weeks taper period. During hospitalization, patients with clinical complications such as ascites, spontaneous bacterial peritonitis, renal dysfunction, overt hepatic encephalopathy (HE) or gastrointestinal bleeding associated with portal hypertension were treated according to current international guidelines (19-22). The Ethics Committee of the Hospital Clinic Barcelona approved the study and all patients gave written informed consent.

\section{Histological, Clinical and Hemodynamic Assessment}

Demographic, clinical, and analytic parameters were collected for creation of a comprehensive database as described previously $(2,23)$. The response to corticosteroid treatment was assessed at 7 days using the Lille Model (24). All liver biopsies in the training set were obtained by transjugular approach within 48 hours of admission. Liver specimens (LS) were formalin-fixed and paraffin-embedded, and slides of $3 \mu \mathrm{m}$ were stained with hematoxylin-eosin and Masson's trichrome. An expert liver pathologist reviewed all biopsies (R.M.) and was blinded. A detailed histological analysis was prospectively performed as follows: (a) degree of hepatocellular damage/ballooning $(0=$ mild and $1=$ marked), (b) presence of Mallory bodies ( $0=$ absent and $1=$ present), (c) degree of polymorphonuclear $(\mathrm{PMN})$ infiltration $(0=$ mild and $1=$ moderate/severe $),(\mathrm{d})$ degree of steatosis $(0=\langle 33 \%, 1=33-66 \%, 2=>66 \%)$, (e) lobular fibrosis $(0=$ no fibrosis or zone 3 , $1=$ fibrosis in zone $2+3$ and $2=$ panlobular fibrosis), (f) fibrosis stage ( $0=$ no fibrosis or portal fibrosis, $1=$ expansive periportal fibrosis, $2=$ bridging fibrosis and cirrhosis $),(\mathrm{g})$ megamitochondria $(0=$ no, $1=y e s)$ and $(\mathrm{h})$ presence and site of bilirubinostasis $(0=$ none, 1 =hepatocellular bilirubinostasis, $2=$ canalicular or ductular bilirubinostasis, $3=$ hepatocellular + canalicular or ductular bilirubinostasis) (25). See Supplementary Data for detailed explanation of histological evaluation. Hepatic hemodynamic assessment was performed within $48 \mathrm{~h}$ of admission. The portal pressure was estimated based on the HVPG, as described in detail previously (26).

\section{Test and Validation Sets}

Five international academic centers contributed 205 consecutive additional patients with alcoholic hepatitis (University Hospital Gasthuisberg, KU Leuven, Belgium; Mayo Clinic, Rochester, MN; Hospital General Universitario Gregorio Marañón, Madrid, Spain; Hôpital Huriez, Lille, France and Royal Free Hospital, London, UK). These patients constituted the test set and validation cohort (see details in Supplementary Methods). All participant centers followed the same inclusion/exclusion criteria outlined above for the training set. In all of these centers, the vast majority of biopsies were performed within $48 \mathrm{~h}$ from admission and 
were processed and analyzed in the same way as in the training set (see Statistical Analysis for details).

The histological assessment of liver specimens was performed by expert liver pathologists. LS from Lille (France) were fixed with alcohol-formalin-acetic acid, the rest of LS where fixed as in the study cohort. Importantly, to ensure a homogeneous histological assessment of all biopsies, a detailed training plan was carried out between the central pathologist (R.M.) and all local pathologists, including teleconferences and presentations describing all histological parameters. To establish an inter-observer agreement between the central pathologist with other participant centers, 71 slide-kits were cross-shared for histological interpretation to determine a kappa coefficient.

\section{Statistical Analysis}

Continuous variables were described as means (95\% confidence interval) or medians (interquartile range). Categorical variables were described by means of counts and percentages. Comparisons between groups were performed using the Student's $t$ test or the Mann-Whitney $U$ test when appropriate. Differences between categorical variables were assessed by the chi-square test or the Fisher exact test. The main endpoint was death at 90 days. Other endpoints were development of clinical complications during hospitalization (i.e. infection) and response to corticosteroids at 7 days.

The initial model was developed in the training set. All histological data were included in the univariate analysis. A logistic regression univariate analysis was used to identify histological features associated with 90-day mortality. Variables that were statistically significant in the univariate model were included in the multivariate logistic regression model and eliminated using backward stepwise elimination. The criterion for retaining predictors was a $\mathrm{p}$ value $<0.10$. The performance of this model was evaluated in the test set and the model was updated by re-estimating the regression coefficients in the training/test set (study cohort). Of note, no further variable selection was performed at this stage. The final coefficients were corrected for optimism with bootstrapping. Finally, the model was converted into a weighted semi-quantitative histological score. At this point the model was "locked". See Supplementary Methods for a detailed explanation of model building. Survival curves were constructed with the Kaplan-Meier method and comparisons were performed using the log-rank test. Calibration of the model in the validation set was assessed by plotting predicted $v s$ observed rate of events (27).

Finally, for inter-observer agreement analysis we determined the kappa coefficient and the weighted kappa coefficient for histological items with multiple categories. To assess if adding analytical variables to the AHHS could improve the prognostic capability for 90-day mortality in patients with $\mathrm{AH}$ we conducted a multivariable logistic regression analysis as follows: AHHS was forced into the model in a first block and clinical variables at admission (e.g. bilirubin, creatinine, age and INR) were included in a second block, and a backwards selection was performed.

Statistical analysis was performed using the SPSS version 12.0 for Windows (SPSS, Inc., Chicago, IL), except for the comparison between ROC curves (concordance [c] statistic 
test), which was performed by the method of Hanley and McNeil using the MedCalc version 9.3.0.0. (Medisoftware, Mariakerke, Belgium) and the kappa analysis which was performed using the Vassar Stats statistical computation website (http://vassarstats.net/).

\section{RESULTS}

\section{Characteristics of the Training Set}

The demographic, clinical, analytical, hemodynamic and histological data of the training set are shown in Table 1. Complications during hospitalization included: 47 (39\%) patients developed a bacterial infection, 22 (18\%) patients developed renal failure, 21 (17\%) patients had gastrointestinal bleeding and $38(31 \%)$ patients had an episode of overt HE. Type of infections during hospitalization were as follows: pulmonary $(26 \%)$, urinary tract $(23 \%)$, soft tissue (8\%), spontaneous bacterial peritonitis (6\%), catheter related infections $(6 \%)$, meningitis (4\%) and others (26\%). The overall 90-day mortality rate was $29 \%$. The main causes of death at 90 days were sepsis $(29 \%)$ and multi-organ failure $(52 \%)$. The presence of AH was confirmed histologically in all patients. The prevalence of different histological findings is shown in Table 1.

\section{Histological Factors Associated with 90-day Survival and Generation of the AHHS score}

We first assessed the association between histological features and short-term survival in the training set. The univariate analysis identified 4 histological features at admission that were significantly associated with 90-day survival. In the multivariate analysis, fibrosis stage, PMN infiltration, type of bilirubinostasis, and presence of megamitochondria independently predicted 90-day survival (Table 2). Of note, the presence of a marked PMN infiltration and presence of megamitochondria were independently associated with a favorable outcome $(\mathrm{OR}=0.13,95 \% \mathrm{CI}-0.03-0.47-$ and $\mathrm{OR}=0.12$, 95\% CI $-0.04-0.36-$, respectively). In contrast, the presence of hepatocellular damage (e.g. Mallory-Denk bodies or hepatocyte ballooning) and the presence/degree of steatosis did not influence short-term prognosis. Representative pictures of the histological features independently associated with 90-day survival are shown in Figure 1 and Figure 2.

The AHHS was generated using the 4 histological features that independently predicted short-term survival as detailed in Supplementary Methods. The final semi-quantitative score is shown in Table 3. Optimal cutoff values to define different severity categories were: 3 and 6 points (sensitivity of $98 \%, 72 \%$ and specificity of $20 \%, 75 \%$, respectively). Using these cutoff values AHHS identified patients with AH with low, moderate, and high 90-day mortality rate. Thirty patients $(14 \%)$ had $\leq 3$ points, 80 patients $(37 \%)$ had $4-5$ points, and 107 patients $(49 \%)$ had $>5$ points. As shown in Figure 3a, these cutoff values strongly discriminate patients with low (97\% survival), intermediate ( $81 \%$ survival), and high risk (49\% survival) of death (Linear Log Rank Test: $\mathrm{p}<.0001$ ), which define patients with mild, moderate and severe $\mathrm{AH}$.

As expected, patients with severe AHHS had a higher rate of life-threating complications (e.g. bacterial infections and renal failure) during hospitalization than patients with moderate 
and mild AHHS (30\% vs 58\% of patients, respectively; $\mathrm{p}=.005)$ In patients with severe AHHS, sepsis was the main cause of death.

We next explored whether the AHHS is also useful in stratifying patients treated with corticosteroids $(n=54)$. When including patients with severe AH treated with corticosteroids, we found that the AHHS accurately stratifies patients into 2 subgroups with different survival at 90 days ( $85 \%$ vs $51 \%$, respectively; $\mathrm{p}=.05$; Supplementary Figure 1).

We finally studied whether the initial AHHS is useful to predict the response to corticosteroids as defined using the Lille Model. We found that patients with a Lille Model below 0.45 (responders to corticosteroids) had the same AHHS than patients with a Lille Model $\geq 0.45$ (non-responders to corticosteroids) (5.2 \pm 2.0 vs. $6.0 \pm 2.0$, respectively; $\mathrm{p}=$. 2). Additionally, we found that none of the individual histological features assessed by AHHS were able to predict the response to corticosteroids at 7 days in these patients. Therefore, histological findings were not useful to predict the response to corticosteroids (Supplementary Table 1).

\section{Validation of the Alcoholic Hepatitis Histological Score (AHHS)}

The baseline characteristics of the test and validation set $(n=205)$ are shown in Supplementary Table 2. Although patients in the external cohorts presented with higher bilirubin levels, MELD and ABIC scores, 90-day survival was comparable with that of the study cohort (69\% vs. 71\%, respectively; $\mathrm{p}=.7$ ). AHHS demonstrated a robust prognostic capability to discriminate into three categories with different outcome at 90 days. AHHS adequately stratified patients with low (0-3 points), moderate (4-5 points) and high (6-9 points) risk of death at 90 -days $(100 \%, 83 \%$ and $64 \%$ survival, respectively; $p=.008$. Figure $3 b)$. Comparison between predicted and observed mortalities in the validation cohort is shown in Supplementary Figure 2.

Importantly, we performed a cross sharing of 71 slide-kits for assessing inter-observer agreement in order to evaluate the reproducibility of the AHHS between our central pathologist and the liver pathologists of participant centers. Analysis of agreement on histological features evaluated by the AHHS showed a moderate agreement for the presence of megamitochondria ( $\mathrm{K}=0.46,95 \% \mathrm{CI}$ : 0.27-0.65), good agreement for fibrosis stage and PMN infiltration ( $\mathrm{K}=0.65,95 \% \mathrm{CI}: 0.36-0.94$ and $\mathrm{K}=0.60$, 95\% CI:0.42-0.78, respectively) and an optimal agreement for the scoring of type of bilirubinostasis with weighted-kappa values of 0.86 (CI 95\%:0.75-0.97). The kappa value for the optimized AHHS was 0.64 (CI 95\%:0.53-0.80).

\section{Histological parameters associated with bacterial infections}

Bacterial infection is one of the main causes of death among patients with AH (28). The development of diagnostic tools capable of identifying patients with early or "occult" bacterial infections will help improve management of these patients. Thus, we analyzed if any of the histological features evaluated by the AHHS were associated with the development of bacterial infections during hospitalization (developed after $48 \mathrm{~h}$ of admission). The multivariate analysis identified the type of bilirubinostasis (OR $1.5795 \%$ 
CI: $1.00-2.47 ; \mathrm{p}=.04$ ) as the only histological feature associated with the development of bacterial infections during hospitalization (Supplementary Table 3). Nearly half of the patients with canalicular/ductular bilirubinostasis or hepatocellular plus canalicular/ductular bilirubinostasis (46\% and 47\%, respectively) developed a bacterial infection during hospitalization (Supplementary Figure 3). Interestingly, we found that increasing levels of serum bilirubin correlated with the progressive degree of bilirubinostasis (Supplementary Figure 4).

\section{Prognostic assessment by combining AHHS with analytical data and sequential use of analytical scoring systems and AHHS}

To evaluate the independent prognostic weight between the AHHS and well-validated scoring systems for AH (e.g. MELD and ABIC scores), a one-step bivariate analysis model including both scores was fitted. We found that AHHS was independently associated with 90-day survival in both the study and validation cohorts (Supplementary Table 4). Next, we studied if adding analytical variables to the AHHS could improve the prognostic capability for 90-day mortality in patients with $\mathrm{AH}$ in the training set. We conducted a multivariable logistic regression analysis as detailed in methods. As shown in Supplementary Table 5, INR ( $\mathrm{p}=.02)$, age $(\mathrm{p}=.003)$ and bilirubin at admission $(\mathrm{p}=.04)$ were the 3 variables with statistical significance in the presence of AHHS. This model combining AHHS and clinical variables had an AUROC of 0.89 (CI 95\%: 0.84-0.95) to predict 90-day survival.

Additionally, a side-by-side comparison between AHHS and the existing prognostic scores (MELD and ABIC score) was performed in the complete cohort $(\mathrm{n}=326)$. The resulting AUROCs were: MELD (0.70), ABIC (0.79) and AHHS (0.73). We did not find significant differences when comparing the AUROCs (AHHS vs MELD: $\mathrm{p}=0.40$ and AHHS $v s$ ABIC: $\mathrm{p}=0.15$. Supplementary Figure 5). Finally, we studied if the sequential use of analytical and histological scores could improve the prognostic assessment of patients with AH. The AHHS did not improve the prognostic assessment in patients with ABIC class A or class $\mathrm{C}$. However, when including patients belonging to ABIC class B, the AHHS was able to discriminate patients into two different risks: patients with low (95\% survival) and moderate risk (70\% survival) of death at 90 days $(\mathrm{p}=.003$; Figure $4 \mathrm{a})$. When analyzing patients with MELD score $\mathcal{2} 1$, the AHHS was able to discriminate two different rates of 90-day survival ( $94 \%$ vs $72 \%$; p=.001; Figure 4b). We found differences in MELD score between patients with AHHS $\leq 5$ and patients with AHHS $\geq 5$ points $(16 \pm 8 v s .23 \pm 9$ points of MELD, respectively; $p<.0001$. Supplementary Figure 6 ). These results suggest that the severity of histological abnormalities is reflected by the analytical parameters present in the MELD score (e.g. bilirubin).

Finally, in order to study prognostic relevance of HVPG either by itself or in combination with the AHHS, we compared the mean HVPG in patients who survived and compared to those who were deceased at 90 days. HVPG was only slightly lower among survivors vs. deceased patients ( $18.5 \pm 6.1 \mathrm{vs}$. $20.8 \pm 6.4 \mathrm{mmHg}$, respectively, $\mathrm{p}=.06$. data not shown). To analyze if HVPG adds prognostic value to AHHS, both variables were included in a logistic regression analysis. HVPG did not show statistical significance (OR:1.08, 95\% CI: 0.99 1.17; $p=.06$, data not shown). Finally, no differences in HVPG measurements were found between patients with definitive AHHS $\geq 5$ and definitive AHHS $<5$ points $(19.0 \pm 6 v s$. 
19.7 $\pm 6 \mathrm{mmHg}$, respectively, $\mathrm{p}=.3$; Supplementary Figure 7). Overall, these results suggest that HVPG measurement is not useful to increase the prognostic performance of AHHS.

\section{DISCUSSION}

The current study has developed a novel histological scoring system capable of predicting short-term survival in patients with AH. Although previous isolated reports had suggested a relationship between single histological parameters and disease outcome (13-17), this is the first systematic investigation including both a study and validation cohort that generates a prognostic histological classification. Such a classification was lacking in the field of AH and can be very useful in stratifying patients for clinical trials, in identifying key disease drivers and to better characterize this devastating disease. Our study was performed in 6 international academic centers from Europe and US and includes 326 patients with biopsyproven AH. Importantly, we performed an extensive measurement of inter-observer agreement, which was lacking for this disease. Our study also defines consistent diagnostic criteria of the main histological findings that define AH. These criteria can be useful to define the parameters that need to be evaluated in the histological assessment of $\mathrm{AH}$.

The stage of fibrosis was identified as an independent predictor of short-term mortality in these patients. This finding is not surprising, given that extensive fibrosis leads to portal hypertension and favors related complications (17). In fact, the degree of portal hypertension has been shown to identify patients with AH and poor outcome (29). In our study, we found that the presence of bridging fibrosis and cirrhosis are associated with poor outcome. Besides increasing the intrahepatic resistance to blood flow, extensive fibrosis suggests more severe underlying liver disease and a poor regenerative response to acute-on-chronic injury. Further studies should investigate the molecular drivers of fibrosis in patients with $\mathrm{AH}$ and whether anti-fibrogenic agents have beneficial effects in these patients.

A striking finding of our study is that the presence and type of bilirubinostasis has a marked influence in short-term mortality. In fact, AH is analytically characterized by profound cholestasis and serum bilirubin levels are one of the most powerful predictors of poor outcome in these patients $(2,5,7)$. Our results confirm previous studies suggesting that bilirubinostasis is one of the most ominous findings in patients with $\mathrm{AH}(15-16)$. The presence of bilirubinostasis may reflect both poor hepatocellular bile transport and difficulties with hepatic bile flow. Ballooned hepatocytes have impaired bile salt secretion and the serum concentration of bile salts is particularly increased in patients with severe $\mathrm{AH}$ (30). Further studies should identify the main molecular drivers of cholestasis in AH and identify therapeutic targets. Interestingly, the presence of bilirubinostasis was associated with the development of bacterial infection and sepsis, which typically cause cholestasis. It is likely that bilirubinostasis reflects poor hepatocellular function which favors bacterial infections and, in turn, bacterial products worsen biliary secretion. In fact, lipopolysaccharide (an important bacterial mediator that is markedly increased in $\mathrm{AH}$ ) down-regulates bile transporters in hepatocytes and causes cholestasis (31-32).

The finding that PMN infiltration and the presence of megamitochondria identifies patients with better outcome confirms previous observations (13-14). Several potential reasons 
potentially explain why the presence of severe PMN infiltration is associated with good outcome. First, patients with more advanced forms of $\mathrm{AH}$ are characterized by poor regenerative response (33) and therefore those presenting with active inflammation may reflect an earlier or less advanced stage of the disease. Second, inflammatory cells, including PMNs, secrete cytokines that stimulate liver regeneration (34). Also, the development of megamitochondria may represent an early stage in hepatocellular damage and has been shown to be associated with a more favorable outcome $(14,35)$. Whether megamitochondria is associated with molecular events that protect hepatocytes from cell death is unknown and deserves further investigation.

The results of our study and the generated AHHS may have important clinical applications. First, the AHHS represents an independent novel tool for prognostic classification in these patients. Identification of patients with different outcome is essential for clinical decisionmaking and to design clinical trials. Of interest, the AHHS allows a more accurate prediction of survival of patients with ABIC class B (moderate risk of death $-25 \%$ of overall mortality), leading to more refined prognostic stratification of these patients. In addition, the AHHS was able to refine the prognostic stratification of patients with MELD $<21$ which are traditionally considered to have a low risk of death (7). In the present study overall mortality of patients with MELD $<21$ was $20.5 \%$ at 90 days. Using a cut-off of 5 points the AHHS was able to define 2 subgroups with different 90-day survival (94\% vs $72 \%$ ). This observation is important since the use of AHHS could stratify patients with a higher risk of mortality among those initially considered low risk. Thus, the sequential use of the ABIC or MELD and AHHS systems allows for precise prognostic assessment of patients with AH with moderate and low risk of death. Second, we identified bilirubinostasis as a useful predictor of infections. This finding should promote clinicians to rule out ongoing infections. Whether the presence of bilirubinostasis by itself justifies the administration of empiric antibiotics is debatable and deserves further study. Finally, by identifying histological parameters leading to increased mortality (ie. extensive fibrosis and bilirubinostasis) we have identified key events that influence disease severity which will certainly help guide development of more accurate animal models of this disease, in order to perform reliable preclinical studies.

It is important to emphasize that the relevance of our novel histological score is not only based on its capability to predict survival. In addition to prognostication, our study reveals which main histological features impact a patient's outcome. Some of the findings of our study will guide future research to develop new therapies for this "orphan" disease that urgently need new approaches. For instance, the finding that PMN infiltration is associated with a more favorable outcome shifts the paradigm in the field of alcoholic hepatitis (AH) and suggests that patients with active inflammation probably have more regenerative potential and are more likely to survive to the episode of AH. Attempts to improve liver regeneration, rather than blocking inflammation, seem more appropriate for this patient population.

Finally, we acknowledge the exclusion of patients without histological criteria of alcoholic steatohepatitis $(n=34)$. Unfortunately, this drawback was due to protocol reasons. The 
prognostic capability of the AHHS in these patients and whether this subset of patients had a more favorable outcome is extremely interesting and deserves future investigation.

\section{Supplementary Material}

Refer to Web version on PubMed Central for supplementary material.

\section{Acknowledgments}

\section{GRANT SUPPORT:}

This work was supported by grants from Fondo de Investigación Sanitaria (FIS PI080237 and FIS PS09/01164 to RB and JC) and by a NIAAA grants 1U01AA021908-01 and P30 DK 034987. JA has a grant from Fundación Banco Bilbao Vizcaya Argentaria (FBBVA) and is enrolled in the Master on Research in Liver Diseases and PhD program of the Universitat de Barcelona. JM receives the grant FPU 11-2607 from Ministerio de Educación de España and is enrolled in the Master on Research in Liver Diseases of the Universitat de Barcelona. JGA has a grand from Instituto de Salud Carlos III (FIS PI11/0883 to JGA), co-financed by FEDER funds (EU, "Una manera de hacer Europa"). Mayo tissue collections were done through P30DK084567 and AA021788.

\section{ABBREVIATIONS}

$\begin{array}{ll}\text { ABIC } & \text { Age-bilirubin-INR-creatinine score } \\ \text { AH } & \text { Alcoholic hepatitis } \\ \text { AHHS } & \text { Alcoholic hepatitis histological score } \\ \text { ALT } & \text { Alanine aminotransferase } \\ \text { AST } & \text { Aspartate aminotransferase } \\ \text { AUROC } & \text { Area under the receiver operating characteristic curve } \\ \text { GGT } & \text { Gamma glutamyl transpeptidase } \\ \text { LS } & \text { Liver specimens } \\ \text { MELD } & \text { Model for end-stage liver disease } \\ \text { OR } & \text { Odds ratio } \\ \text { PMN } & \text { Polymorphonuclear }\end{array}$

\section{References}

1. Lucey MR, Mathurin P, Morgan TR. Alcoholic hepatitis. N Engl J Med. 2009; 360:2758-2769. [PubMed: 19553649]

2. Dominguez M, Rincon D, Abraldes JG, et al. A new scoring system for prognostic stratification of patients with alcoholic hepatitis. Am J Gastroenterol. 2008; 103:2747-56. [PubMed: 18721242]

3. European Association For The Study Of The Liver. EASL Clinical Practical Guidelines: Management of Alcoholic Liver Disease. J Hepatol. 2012; 57:399-420. [PubMed: 22633836]

4. Shah VH. Alcoholic liver disease: the buzz may be gone, but the hangover remains. Hepatology. 2010; 51:1483-4. [PubMed: 20432249]

5. Maddrey WC, Boitnott JK, Bedine MS, et al. Corticosteroid therapy of alcoholic hepatitis. Gastroenterology. 1978; 75:193-199. [PubMed: 352788] 
6. Forrest EH, Evans CD, Stewart S, et al. Analysis of factors predictive of mortality in alcoholic hepatitis and derivation and validation of the Glasgow alcoholic hepatitis score. Gut. 2005; 54:1174-1179. [PubMed: 16009691]

7. Dunn W, Jamil LH, Brown LS, et al. MELD accurately predicts mortality in patients with alcoholic hepatitis. Hepatology. 2005; 41:353-358. [PubMed: 15660383]

8. Kleiner DE, Brunt EM, Van Natta M, et al. Design and validation of a histological scoring system for nonalcoholic fatty liver disease. Hepatology. 2005; 41:1313-21. [PubMed: 15915461]

9. Bedossa P, Poynard T. An algorithm for the grading of activity in chronic hepatitis C. The METAVIR Cooperative Study Group. Hepatology. 1996; 24:289-93. [PubMed: 8690394]

10. Ludwig J, Dickson ER, McDonald GS. Staging of chronic nonsuppurative destructive cholangitis (syndrome of primary biliary cirrhosis). Virchows Arch A Pathol Anat Histol. 1978; 22;379:10312.

11. Ludwig J, Barham SS, LaRusso NF. Morphologic features of chronic hepatitis associated with primary sclerosing cholangitis and chronic ulcerative colitis. Hepatology. 1981; 1:632-40. [PubMed: 7308996]

12. Hennes EM, Zeniya M, Czaja AJ, et al. Simplified criteria for the diagnosis of autoimmune hepatitis. Hepatology. 2008 Jul; 48(1):169-76. [PubMed: 18537184]

13. Mathurin P, Duchatelle V, Ramond MJ, et al. Survival and prognostic factors in patients with severe alcoholic hepatitis treated with prednisolone. Gastroenterology. 1996; 110:1847-53. [PubMed: 8964410]

14. Chedid A, Mendenhall CL, Tosch T, et al. Significance of megamitochondria in alcoholic liver disease. Gastroenterology. 1986; 90:1858-64. [PubMed: 3699404]

15. Katoonizadeh A, Laleman W, Verslype C, et al. Early features of acute-on-chronic alcoholic liver failure: a prospective cohort study. Gut. 2010; 59:1561-9. [PubMed: 20675694]

16. Spahr L, Rubbia-Brandt L, Genevay M, et al. Early liver biopsy, intraparenchymal cholestasis, and prognosis in patients with alcoholic steatohepatitis. BMC Gastroenterol. 2011; 11:115. [PubMed: 22035247]

17. Mookerjee RP, Lackner C, Stauber R, et al. The role of liver biopsy in the diagnosis and prognosis of patients with acute deterioration of alcoholic cirrhosis. J Hepatol. 2011; 55:1103-11. [PubMed: 21376092]

18. Colmenero J, Bataller R, Sancho-Bru P, et al. Hepatic expression of candidate genes in patients with alcoholic hepatitis: correlation with disease severity. Gastroenterology. 2007; 132:687-97. [PubMed: 17258719]

19. Garcia-Tsao G, Sanyal AJ, Grace ND, et al. Prevention and management of gastroesophageal varices and variceal hemorrhage in cirrhosis. Hepatology. 2007; 46:922-38. [PubMed: 17879356]

20. Moore KP, Wong F, Ginès P, et al. The management of ascites in cirrhosis: report on the consensus conference of the International Ascites Club. Hepatology. 2003; 38:258-266. [PubMed: 12830009]

21. B A Runyon and Practice Guidelines Committee, American Association for the Study of Liver Diseases (AASLD). Management of adult patients with ascites due to cirrhosis. Hepatology. 2004; 39:841-855. [PubMed: 14999706]

22. Ferenci P, Lockwood A, Mullen K, et al. Hepatic encephalopathy--definition, nomenclature, diagnosis, and quantification: final report of the working party at the 11th World Congresses of Gastroenterology, Vienna, 1998. Hepatology. 2002; 35:716-21. [PubMed: 11870389]

23. Altamirano J, Fagundes C, Dominguez M, et al. Acute kidney injury is an early predictor of mortality for patients with alcoholic hepatitis. Clin Gastroenterol Hepatol. 2012; 10:65-71. [PubMed: 21946124]

24. Louvet A, Naveau S, Abdelnour M, et al. The Lille model: a new tool for therapeutic strategy in patients with severe alcoholic hepatitis treated with steroids. Hepatology. 2007; 45:1348-54. [PubMed: 17518367]

25. Desmet VJ. Histopathology of cholestasis. Verh Dtsch Ges Pathol. 1995; 79:233-40. [PubMed: 8600686]

26. Bosch J, Abraldes JG, Berzigotti A, et al. The clinical use of HVPG measurements in chronic liver disease. Nat Rev Gastroenterol Hepatol. 2009; 6:573-82. [PubMed: 19724251] 
27. Micheel, C. IOM (Institute of Medicine). Evolution of Translational Omics: Lessons Learned and the Path Forward. Washington, DC: The National Academies Press; 2012. p. 43-44.

28. Louvet A, Wartel F, Castel H, et al. Infection in patients with severe alcoholic hepatitis treated with steroids: early response to therapy is the key factor. Gastroenterology. 2009; 137:541-8. [PubMed: 19445945]

29. Rincon D, Lo Iacono O, Ripoll C, et al. Prognostic value of hepatic venous pressure gradient for in-hospital mortality of patients with severe acute alcoholic hepatitis. Aliment Pharmacol Ther. 2007; 25:841-8. [PubMed: 17373923]

30. Trinchet JC, Gerhardt MF, Balkau, et al. Serum bile acids and cholestasis in alcoholic hepatitis. Relationship with usual liver tests and histological features. J Hepatol. 1994; 21:235-40. [PubMed: 7989715]

31. Elferink MG, Olinga P, Draaisma AL, et al. LPS-induced downregulation of MRP2 and BSEP in human liver is due to a posttranscriptional process. Am J Physiol Gastrointest Liver Physiol. 2004; 287:G1008-16. [PubMed: 15205115]

32. Lefkowitch JH. Bile ductular cholestasis: an ominous histopathologic sign related to sepsis and "cholangitis lenta". Hum Pathol. 1982; 13:19-24. [PubMed: 7076191]

33. Sancho-Bru P, Altamirano J, Rodrigo-Torres D, et al. Liver progenitor cell markers correlate with liver damage and predict short-term mortality in patients with alcoholic hepatitis. Hepatology. 2012; 55:1931-41. [PubMed: 22278680]

34. Taïeb J, Delarche C, Paradis V, et al. Polymorphonuclear neutrophils are a source of hepatocyte growth factor in patients with severe alcoholic hepatitis. J Hepatol. 2002; 36:342-8. [PubMed: 11867177]

35. Stewart RV, Dincsoy HP. The significance of giant mitochondria in liver biopsies as observed by light microscopy. Am J Clin Pathol. 1982; 78:293-8. [PubMed: 6180628] 


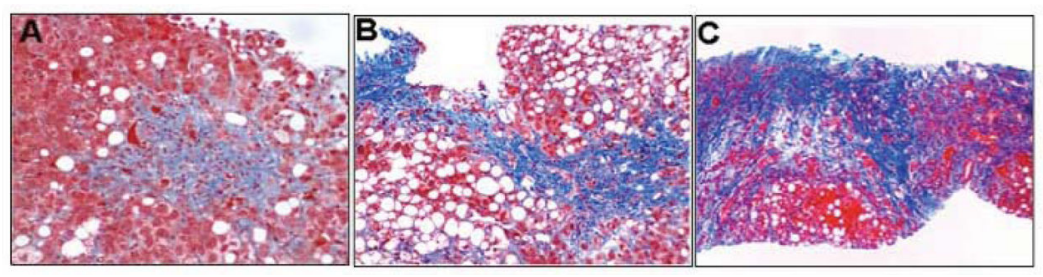

Figure 1.

Histological Features Independently Associated with 90-day Survival Included in the Alcoholic Hepatitis Histological Score (AHHS). A, B and C) Degree of fibrosis (Masson's Trichrome staining); portal fibrosis, expansive areas of liver fibrosis and cirrhosis, respectively. Photomicrographs A, B and C: Hematoxylin and eosin staining. 


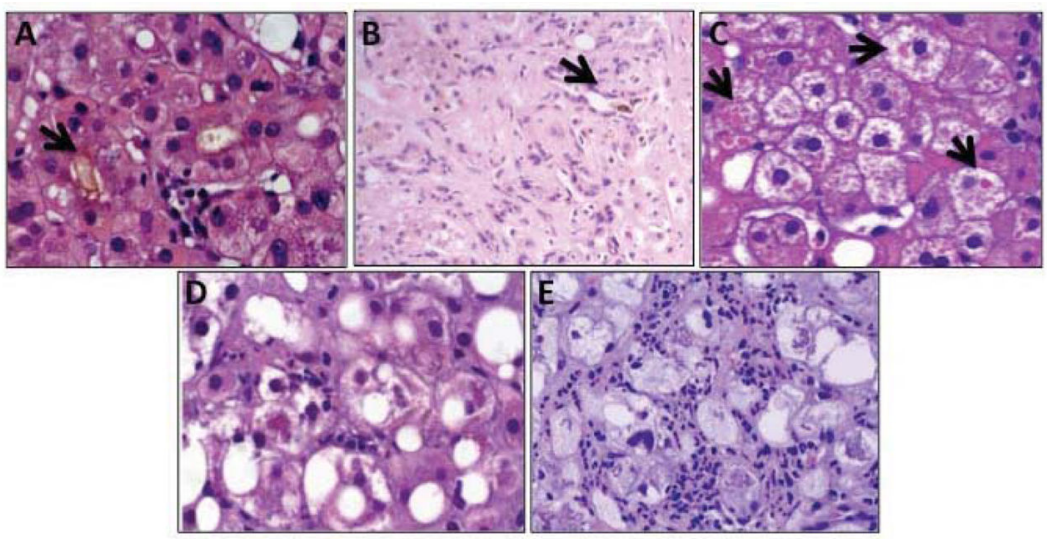

Figure 2.

Histological Features Independently Associated with 90-day Survival Included in the Alcoholic Hepatitis Histological Score (AHHS). A) Hepatocellular and canalicular bilirubinostasis (arrow). B) Ductular bilirubinostasis (arrow). C) Megamitochondria (arrows). D and E) Mild and severe PMN infiltration, respectively. Photomicrographs A-D and E: Hematoxylin and eosin staining; PNM: Polymorphonuclear cells. 

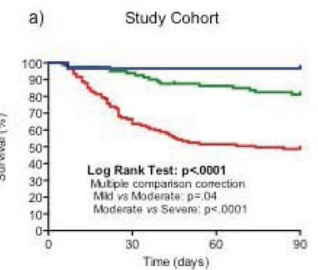

$\begin{array}{lllll}\text { Mad (0-3) } & 30 & 29 & 29 & 29 \\ \text { Moderate (4-5) } & 80 & 75 & 6 B & 65\end{array}$

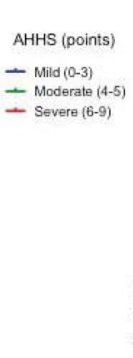

AHHS (points)

- Midd (0-3)

Severe (6-9)

Severe (6-9) 102

Figure 3.

Three-month Survival Probability of Patients with Alcoholic Hepatitis According to the Alcoholic Hepatitis Histological Score (AHHS) in the study (a) and validation (b) cohorts. 

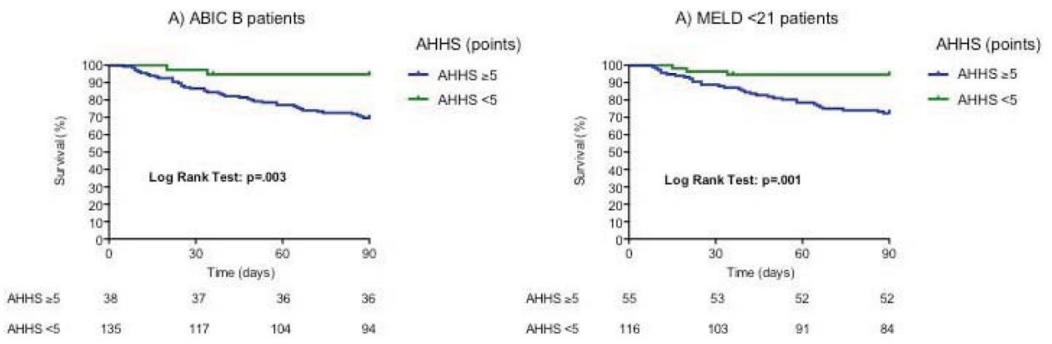

Figure 4.

a) Three-month survival probability in patients with ABIC B according to the Alcoholic Hepatitis Histological Score. a) Three-month survival probability in patients with MELD $<21$ according to the Alcoholic Hepatitis Histological Score 


\section{Table 1}

Baseline Demographic, Clinical, Hemodynamic and Histological Characteristics of Patients with Alcoholic Hepatitis in the Training Cohort $(\mathrm{n}=121)$

\begin{tabular}{|c|c|}
\hline Characteristics & Median (25-75 IQR) \\
\hline Age (y) & $49(41-54)$ \\
\hline Male n (\%) & $78(67)$ \\
\hline Alcohol intake (g/day) & $100(100-120)$ \\
\hline Corticosteroids n $(\%)$ & $54(45)$ \\
\hline Biopsy Length (cm) & $0.6(0.4-1.2)$ \\
\hline \multicolumn{2}{|c|}{ Clinical decompensations at admission } \\
\hline Ascites n (\%) & $82(68)$ \\
\hline Variceal bleeding $\mathrm{n}(\%)$ & $26(21)$ \\
\hline Encephalopathy n (\%) & $17(14)$ \\
\hline Infection $\mathrm{n}(\%)$ & $18(15)$ \\
\hline \multicolumn{2}{|c|}{ Laboratory and hemodynamic parameters } \\
\hline Hemoglobin $(\mathrm{g} / \mathrm{dL})$ & $11(9-12)$ \\
\hline Leukocyte count $\times 10^{9} / \mathrm{L}$ & $8.9(6.3-13.9)$ \\
\hline Platelet count $\times 10^{9} / \mathrm{L}$ & $121(71-175)$ \\
\hline $\operatorname{AST}(\mathrm{U} / \mathrm{L})$ & $125(82-188)$ \\
\hline ALT (U/L) & $51(35-71)$ \\
\hline Serum $\mathrm{Na}(\mathrm{mmol} / \mathrm{L})$ & $134(130-137)$ \\
\hline Serum albumin $(\mathrm{g} / \mathrm{dL})$ & $2.7(2.3-3.0)$ \\
\hline Serum creatinine $(\mathrm{mg} / \mathrm{dL})$ & $0.8(0.60-1.0)$ \\
\hline Serum bilirubin (mg/dL) & $9.7(4.3-17.7)$ \\
\hline International normalized ratio & $1.6(1.4-1.9)$ \\
\hline $\operatorname{HVPG}(\mathrm{mmHg})^{*}$ & $20(15-24)$ \\
\hline \multicolumn{2}{|c|}{ Alcoholic hepatitis severity scores at admission } \\
\hline MELD score & $18(12-22)$ \\
\hline ABIC score & $7.3(6.6-8.4)$ \\
\hline \multicolumn{2}{|l|}{ ABIC class $\mathrm{n}(\%)$} \\
\hline $\mathrm{A}(<6.71)$ & $33(27)$ \\
\hline B $(6.7-8.99)$ & $68(56)$ \\
\hline $\mathrm{C}\left(\otimes^{2}\right)$ & $20(17)$ \\
\hline \multicolumn{2}{|c|}{ Histological features at admission n (\%) } \\
\hline \multicolumn{2}{|l|}{ Fibrosis stage } \\
\hline No Fibrosis or Portal fibrosis & $3(2)$ \\
\hline Expansive fibrosis & $19(16)$ \\
\hline Bridging fibrosis or Cirrhosis & $99(82)$ \\
\hline \multicolumn{2}{|l|}{ Steatosis } \\
\hline$<33 \%$ & $39(32)$ \\
\hline $33-66 \%$ & $35(29)$ \\
\hline$>66 \%$ & $47(39)$ \\
\hline
\end{tabular}




\begin{tabular}{lc}
\hline Characteristics & Median (25-75 IQR) \\
\hline Mallory bodies & $4(3)$ \\
No or Occasional & $117(97)$ \\
Marked & \\
Bilirubinostasis & $37(31)$ \\
No & $14(12)$ \\
Hepatocellular & $32(26)$ \\
Canalicular and/or ductular & $38(31)$ \\
Hepatocellular plus canalicular and/or ductular & \\
Ballooning & $48(40)$ \\
Occasional & $73(60)$ \\
Marked & \\
PMN infiltration & $81(67)$ \\
No/Mild & $40(33)$ \\
Severe & \\
Megamitochondria & $30(25)$ \\
No & $91(75)$ \\
Yes &
\end{tabular}

Abbreviations: IQR, interquartile range; AST, aspartate aminotransferase level; ALT, alanine aminotransferase level; HVPG, hepatic venous pressure gradient; MELD, model for end-stage liver disease; ABIC, age-bilirubin-INR-creatinine score; PMN, polymorphonuclears

* Data from 112 patients 


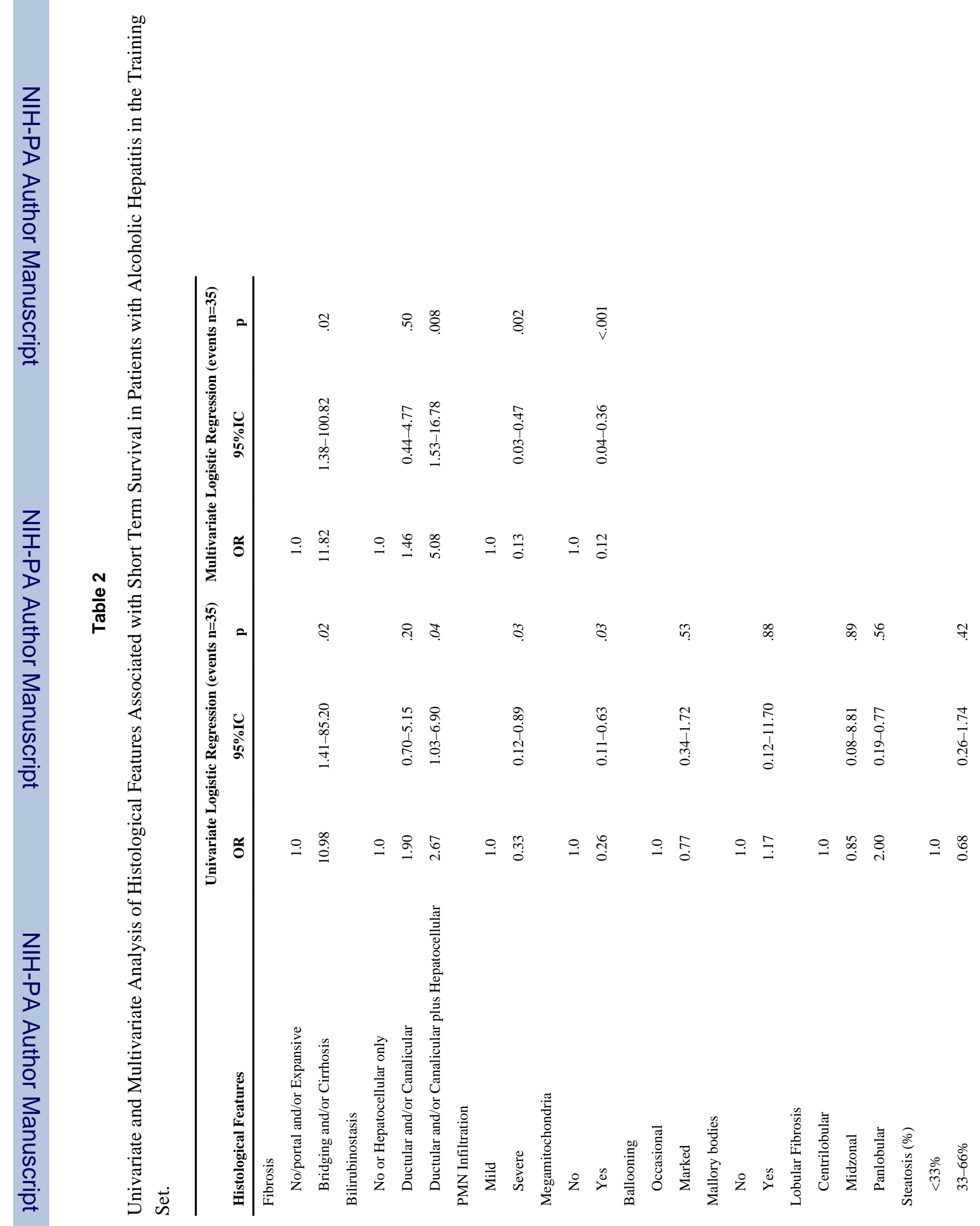




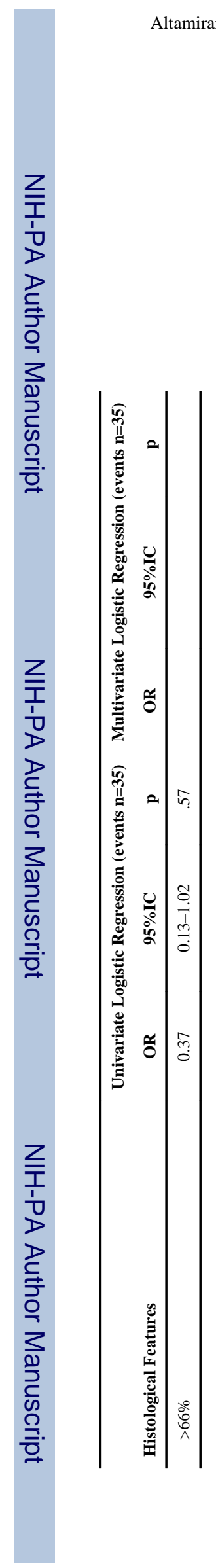

Page 21 
Table 3

Alcoholic Hepatitis Histological Score (AHHS) for Prognostic Stratification of Alcoholic Hepatitis

\begin{tabular}{lcl}
\hline & Points & \\
\hline Fibrosis stage & & AHHS categories (0-9 points) \\
None Fibrosis or Portal fibrosis & 0 & Mild : 0-3 \\
Expansive fibrosis & 0 & Intermediate: 4-5 \\
Bridging fibrosis or Cirrhosis & +3 & Severe: 6-9 \\
Bilirubinostasis & & \\
No & 0 & \\
Hepatocellular only & 0 & \\
Canalicular or ductular & +1 & \\
Canalicular or ductular plus Hepatocellular & +2 & \\
PMN infiltration & \\
No/Mild & +2 \\
Severe PMN Infiltration & 0 \\
Megamitochondria & \\
No Megamitochondria & +2 \\
Megamitochondria & 0 \\
\hline
\end{tabular}

Abreviations: PMN: polymorphonuclears.

Note: Histological features included in the AHHS where product of the multivariate logistic regression analysis (Table 2). Weighting of each histological feature was based in the odds ratio of the updated model (training plus test set samples. See model building in Supplementary Data). 